\title{
Stationary Distribution and Extinction of a Stochastic Viral Infection Model
}

\author{
Yan Wang ${ }^{1}$ and Daqing Jiang ${ }^{1,2}$ \\ ${ }^{1}$ College of Science, China University of Petroleum (East China), Qingdao, Shandong 266580, China \\ ${ }^{2}$ Nonlinear Analysis and Applied Mathematics (NAAM) Research Group, Department of Mathematics, \\ Faculty of Science, King Abdulaziz University, Jeddah 121589, Saudi Arabia \\ Correspondence should be addressed to Daqing Jiang; daqingjiang2010@hotmail.com
}

Received 22 July 2017; Revised 2 September 2017; Accepted 18 September 2017; Published 22 October 2017

Academic Editor: Manuel De la Sen

Copyright (C) 2017 Yan Wang and Daqing Jiang. This is an open access article distributed under the Creative Commons Attribution License, which permits unrestricted use, distribution, and reproduction in any medium, provided the original work is properly cited.

\begin{abstract}
We present a kind of stochastic viral infection model with or without a loss term in the free virus equation. We obtain critical condition to ensure the existence of the unique stationary distribution by constructing Lyapunov functions. We also obtain the sufficient conditions for the extinction of the virus by the comparison theorem of stochastic differential equation and law of large numbers. We give a unified method to systematically analyze such three-dimensional stochastic viral infection model. Furthermore, numerical simulations are carried out to examine the effect of white noises on model behavior. We investigate the fact that the small magnitudes of white noises can sustain the irregular recurrence of healthy target cells and virions, while the big ones may contribute to viral clearance.
\end{abstract}

\section{Introduction}

More and more attention has been paid to the mathematical modelling of virus (such as HIV and hepatitis B/C virus) infection. The existing viral models mainly contain three compartments: target cells, actively infected cells, and matured virions; see, for example, [1-10] and the references cited therein. These models are all derived from the basic viral dynamics models [1-3]. However, all these viral models are deterministic models and do not take into account the stochastic fluctuation. Previously, through experimental data, Singh et al. have investigated the fact that the presence of random noise in gene expression may lead to an increase of variability in HIV early gene products, which may seriously affect the fate of the virus between replication and latency [11]. Thus, Mao et al. have shown that, from the perspective of mathematical analysis, a small amount of random fluctuation can inhibit a potential population explosion [12]. Hence, including the effect of random noise in viral dynamics model may play an important role in the development of a better understanding of the disease.

With the development of stochastic epidemic models of between-host transmission [13-17], the stochastic models of within-host viral infection have also been developed in recent years [18-23]. For most of models, these have been considered as the stochastic effects of the model parameters [19-21,23], but these models did not consider the loss term of virus when a free virus enters the target cell. This term is often neglected even though in the deterministic models, because it is typically very small and can be absorbed into the virus clearance term. However, recent studies have showed that the inclusion of this term may have a major impact on the basic reproductive number and viral dynamics under certain parameter sets $[4,5,24]$. It may also affect the probability of extinction of an initial viral load through Monte Carlo approaches $[25,26]$. Therefore, it is more reasonable to include the virus loss term in stochastic viral infection model, which would have further influence on the model behavior. Few stochastic viral infection models $[18,22]$ have included the virus loss term, while the theoretical results are needed to be complete.

In this paper, we extend the classical deterministic viral infection model with or without the virus loss term to a stochastic model of parameter fluctuations. The rest of this paper is organized as follows. In the next section, we formulate our model and show the existence and uniqueness 
of the global positive solution. In Section 3, by constructing suitable Lyapunov functions, the existence of a unique ergodic stationary distribution is derived. We establish the sufficient conditions for the extinction of the virus in Section 4. In Section 5, by employing numerical simulations, we investigate how the white noises affect the model dynamic behavior under realistic parameters. Finally, we conclude our results and give future work.

\section{Model Derivation}

The basic viral infection model [1-3] with or without a loss term in free virus equation can be described by the following ordinary differential equations:

$$
\begin{aligned}
\frac{d T}{d t} & =\lambda-d T-k T V, \\
\frac{d T^{*}}{d t} & =k T V-\delta T^{*}, \\
\frac{d V}{d t} & =N \delta T^{*}-c V-i k T V, \quad i=0 \text { or } 1,
\end{aligned}
$$

where $T, T^{*}$, and $V$ denote the concentrations of healthy target cells, actively infected target cells, and infectious virus particles, respectively. Parameter $\lambda$ is the constant input rate, $k$ presents the viral infection rate between healthy target cells and infectious virus. Parameters $d$ and $\delta$ are the death rates of the healthy and infected target cells, respectively. $N$ is the average number of virus particles produced by an infected target cell and $c$ is the clearance rate of the infectious virus. To reflect the model inclusion or omission of a loss term in free virus equation, we use a term $-i k V T$ in the third equation and $i$ is a fixed constant. When $i=0$, it means that model (1) ignores the loss term $k V T$ due to infection; when $i=1$, it means that model (1) includes the loss term $k V T$ due to infection.

In literature [4], de Leenheer and Smith have obtained the theoretical results of (1). System (1) always has a diseasefree steady state $E_{0}\left(T_{0}, 0,0\right)$, where $T_{0}=\lambda / d$. The basic reproduction number is given by

$$
R_{0, i}=\frac{k N T_{0}}{c+i k T_{0}}, \quad i=0 \text { or } 1 .
$$

System (1) has the following main results:

(i) If $R_{0}<1$, the disease-free steady state $E_{0}$ is globally asymptotically stable.

(ii) If $R_{0}>1$, then a chronic disease steady state exists which is globally asymptotically stable, and the disease-free steady state $E_{0}$ is unstable.

In this paper, considering random fluctuation, we assume that the stochastic fluctuation is the white noise type, which is a linear perturbation corresponding to the rate of change for each population. The stochastic differential model can be written as follows:

$$
\begin{array}{rl}
d T & =(\lambda-d T-k T V) d t+\sigma_{1} T d B_{1}(t), \\
d T^{*} & =\left(k T V-\delta T^{*}\right) d t+\sigma_{2} T^{*} d B_{2}(t), \\
d V & =\left(N \delta T^{*}-c V-i k T V\right) d t+\sigma_{3} V d B_{3}(t), \\
i & i=0 \text { or } 1,
\end{array}
$$

where $B_{i}(t)$ are independent standard Brownian motions with $B_{i}(0)=0$ and $\sigma_{i}^{2}>0$ denote the intensities of the white noise, $i=1,2,3$. The other parameters are the same as those in system (1).

In this paper, we assume $\left(\Omega, \mathscr{F},\left\{\mathscr{F}_{t}\right\}_{t \geq 0}, \mathbb{P}\right)$ as a complete probability space with a filtration $\left\{\mathscr{F}_{t}\right\}_{t \geq 0}$ satisfying the usual conditions (i.e., it is increasing and right continuous while $\mathscr{F}_{0}$ contains all $\mathbb{P}$-null sets $)$, and $B_{i}(t)(i=1,2,3)$ are defined on this complete probability space. We also let $\mathbb{R}_{+}^{3}=\{x=$ $\left.\left(x_{1}, x_{2}, x_{3}\right) \in \mathbb{R}^{3}: x_{i}>0, i=1,2,3\right\}$. We use $a \wedge b$ to denote $\min \{a, b\}$ and use $a \vee b$ to denote $\max \{a, b\}$.

According to the theory of Arnold [27] and Mao et al. [12], we show the existence and uniqueness of the solution of system (3) in the following Lemma. See Theorem 4.1 of [18] for the detailed proof.

Lemma 1. System (3) has a unique and positive solution $\left(T(t), T^{*}(t), V(t)\right)$ with the initial value $\left(T(0), T^{*}(0), V(0)\right)$ for all $t \geq 0$, and the solution will remain in $\mathbb{R}_{+}^{3}$ with probability one, namely, $\left(T(t), T^{*}(t), V(t)\right) \in \mathbb{R}_{+}^{3}$ for all $t \geq 0$ almost surely (a.s.).

\section{Stationary Distribution}

In this section, by using the theory of Hasminskii [28], we prove the existence of a unique ergodic stationary distribution, which indicates that the virus is prevalent.

Denote

$$
\sigma^{2}=\sigma_{1}^{2} \vee \sigma_{2}^{2} \vee \sigma_{3}^{2}
$$

and define the critical condition

$$
\begin{aligned}
& R_{0, i}^{s} \\
& =\frac{\lambda k N \delta}{\left(d+(1 / 2) \sigma_{1}^{2}\right)\left(\delta+(1 / 2) \sigma_{2}^{2}\right)\left(c+i k T_{0}+(1 / 2) \sigma_{3}^{2}\right)}, \\
& i=0 \text { or } 1 .
\end{aligned}
$$

Theorem 2. If $R_{0, i}^{s}>1$, then system (3) has a unique stationary distribution $\mu(\cdot)$ and it has the ergodic property.

Proof. We firstly verified the establishment of condition (H1) in Lemma A.1 in Appendix. For system (3), the diffusion matrix is

$$
A=\left[\begin{array}{ccc}
\sigma_{1}^{2} T^{2} & 0 & 0 \\
0 & \sigma_{2}^{2}\left(T^{*}\right)^{2} & 0 \\
0 & 0 & \sigma_{3}^{2} V^{2}
\end{array}\right]
$$


Choosing $E=\min _{\left(T, T^{*}, V\right) \in \bar{D}_{k} \subset \mathbb{R}_{+}^{3}}\left\{\sigma_{1}^{2} T^{2}, \sigma_{2}^{2}\left(T^{*}\right)^{2}, \sigma_{3}^{2} V^{2}\right\}$, we have

$$
\begin{aligned}
& \sum_{i, j=1}^{3} a_{i j}\left(T, T^{*}, V\right) \xi_{i} \xi_{j}= \sigma_{1}^{2} T^{2} \xi_{1}^{2}+\sigma_{2}^{2}\left(T^{*}\right)^{2} \xi_{2}^{2} \\
&+\sigma_{3}^{2} V^{2} \xi_{3}^{2} \geq E|\xi|^{2}, \\
&\left(T, T^{*}, V\right) \in \bar{D}_{k}, \quad \xi=\left(\xi_{1}, \xi_{2}, \xi_{3}\right) \in \mathbb{R}_{+}^{3},
\end{aligned}
$$

where $\bar{D}_{k}=[1 / k, k] \times[1 / k, k] \times[1 / k, k]$, and $k$ is a sufficiently large integer; then condition (H1) in Lemma A.1 is satisfied.

Next, we show the validity of condition (H2) in Lemma A.1 in Appendix. Now, construct a $C^{2}$-function $W_{1}$ : $\mathbb{R}_{+}^{3} \rightarrow \mathbb{R}$

$$
W_{1}=-\ln T-c_{1} \ln T^{*}-c_{2}\left(\ln V-i \frac{k T}{d}\right),
$$

where $i=0$ or $1 ; c_{1}$ and $c_{2}$ are positive constants which will be determined later. Applying Itô's formula [29] to $W_{1}$ yields

$$
\begin{aligned}
L\left(-\ln V+i \frac{k T}{d}\right)= & -\frac{N \delta T^{*}}{V}+c+i \frac{k \lambda}{d}-i \frac{k^{2} V T}{d} \\
& +\frac{1}{2} \sigma_{3}^{2} \\
\leq & -\frac{N \delta T^{*}}{V}+c+i k T_{0}+\frac{1}{2} \sigma_{3}^{2},
\end{aligned}
$$

and we should mention that $T_{0}=\lambda / d$. Thus,

$$
\begin{aligned}
L W_{1} \leq & -\frac{\lambda}{T}-c_{1} \frac{k T V}{T^{*}}-c_{2} \frac{N \delta T^{*}}{V}+d+\frac{1}{2} \sigma_{1}^{2} \\
& +c_{1}\left(\delta+\frac{1}{2} \sigma_{2}^{2}\right)+c_{2}\left(c+i k T_{0}+\frac{1}{2} \sigma_{3}^{2}\right)+k V \\
\leq & -3 \sqrt[3]{\lambda k N \delta c_{1} c_{2}}+c_{1}\left(\delta+\frac{1}{2} \sigma_{2}^{2}\right) \\
& +c_{2}\left(c+i k T_{0}+\frac{1}{2} \sigma_{3}^{2}\right)+\left(d+\frac{1}{2} \sigma_{1}^{2}\right)+k V .
\end{aligned}
$$

Let

$$
\begin{aligned}
c_{1}\left(\delta+\frac{1}{2} \sigma_{2}^{2}\right) & =c_{2}\left(c+i k T_{0}+\frac{1}{2} \sigma_{3}^{2}\right) \\
& =\frac{\lambda k N \delta}{\left(\delta+(1 / 2) \sigma_{2}^{2}\right)\left(c+i k T_{0}+(1 / 2) \sigma_{3}^{2}\right)},
\end{aligned}
$$

and calculate that

$$
\begin{aligned}
& c_{1}=\frac{\lambda k N \delta}{\left(\delta+(1 / 2) \sigma_{2}^{2}\right)^{2}\left(c+i k T_{0}+(1 / 2) \sigma_{3}^{2}\right)}, \\
& c_{2}=\frac{\lambda k N \delta}{\left(\delta+(1 / 2) \sigma_{2}^{2}\right)\left(c+i k T_{0}+(1 / 2) \sigma_{3}^{2}\right)^{2}} .
\end{aligned}
$$

Hence,

$$
\begin{aligned}
L W_{1} \leq & -\frac{\lambda k N \delta}{\left(\delta+(1 / 2) \sigma_{2}^{2}\right)\left(c+i k T_{0}+(1 / 2) \sigma_{3}^{2}\right)} \\
& +\left(d+\frac{1}{2} \sigma_{1}^{2}\right)+k V \\
\leq & -\left(d+\frac{1}{2} \sigma_{1}^{2}\right)\left(R_{0, i}^{s}-1\right)+k V=-\beta+k V,
\end{aligned}
$$

where

$$
\begin{aligned}
R_{0, i}^{s} & =\frac{\lambda k N \delta}{\left(d+(1 / 2) \sigma_{1}^{2}\right)\left(\delta+(1 / 2) \sigma_{2}^{2}\right)\left(c+(1 / 2) \sigma_{3}^{2}\right)}, \\
\beta & =\left(d+\frac{1}{2} \sigma_{1}^{2}\right)\left(R_{0, i}^{s}-1\right) .
\end{aligned}
$$

Define a $C^{2}$-function $W: \mathbb{R}_{+}^{3} \rightarrow \mathbb{R}$, in the following form:

$$
W\left(T, T^{*}, V\right)=M W_{1}+W_{2}+W_{3}+W_{4},
$$

where

$$
\begin{aligned}
& W_{2}=-\ln T, \\
& W_{3}=-\ln T^{*}, \\
& W_{4}=\frac{1}{1+\theta}\left(T+T^{*}+\frac{V}{2 N}\right)^{1+\theta},
\end{aligned}
$$

where $0<\theta<\min \left\{1,\left(1 / 2 \sigma^{2}\right)(2 d \wedge \delta \wedge 2 c)\right\}$. Select a suitable constant $M>0$ satisfying the following condition:

$$
-M \beta+C \leq-2,
$$

where

$$
\begin{aligned}
C & =\sup _{\left(T, T^{*}, V\right) \in \mathbb{R}_{+}^{3}}\left\{-\frac{d}{2} T^{1+\theta}-\frac{\delta}{4}\left(T^{*}\right)^{1+\theta}-\frac{c}{2}\left(\frac{V}{2 N}\right)^{1+\theta}\right. \\
& \left.+E+d+\delta+\frac{1}{2} \sigma_{1}^{2}+\frac{1}{2} \sigma_{2}^{2}\right\}<\infty .
\end{aligned}
$$

It is easy to check that

$$
\liminf _{n \rightarrow \infty,\left(T, T^{*}, V\right) \in \mathbb{R}_{+}^{3} \backslash D_{k}} W\left(T, T^{*}, V\right)=+\infty,
$$

where $D_{k}=(1 / k, k) \times(1 / k, k) \times(1 / k, k)$. Furthermore, $W\left(T, T^{*}, V\right)$ is a continuous function. Hence $W\left(T, T^{*}, V\right)$ must have a minimum point $\left(\bar{T}_{0}, \bar{T}^{*}{ }_{0}, \bar{V}_{0}\right)$ in the interior of $\mathbb{R}_{+}^{3}$. Then we define a nonnegative $C^{2}$-function $\bar{W}: \mathbb{R}_{+}^{3} \rightarrow \mathbb{R}$ as follows:

$$
\bar{W}\left(T, T^{*}, V\right)=W\left(T, T^{*}, V\right)-W\left(\bar{T}_{0},{\overline{T^{*}}}_{0}, \bar{V}_{0}\right) .
$$


Applying Itô formula,

$$
\begin{aligned}
L W_{2} & =-\frac{\lambda}{T}+d+k V+\frac{1}{2} \sigma_{1}^{2} \\
L W_{3} & =-\frac{k T V}{T^{*}}+\delta+\frac{1}{2} \sigma_{2}^{2} \\
L W_{4} & =\left(T+T^{*}+\frac{V}{2 N}\right)^{\theta} \\
\cdot & \left(\lambda-d T-\frac{\delta T^{*}}{2}-\frac{c V}{2 N}-i \frac{k T V}{2 N}\right) \\
+ & \frac{\theta}{2}\left(T+T^{*}+\frac{V}{2 N}\right)^{\theta-1} \\
\cdot & {\left[\sigma_{1}^{2} T^{2}+\sigma_{2}^{2}\left(T^{*}\right)^{2}+\sigma_{3}^{2}\left(\frac{V}{2 N}\right)^{2}\right] } \\
& \leq\left(T+T^{*}+\frac{V}{2 N}\right)^{\theta}\left(\lambda-d T-\frac{\delta T^{*}}{2}-\frac{c V}{2 N}\right)^{2} \frac{\theta}{2} \\
\cdot & \sigma^{2}\left(T+T^{*}+\frac{V}{2 N}\right)^{\theta-1}\left[T^{2}+\left(T^{*}\right)^{2}+\left(\frac{V}{2 N}\right)^{2}\right] \\
& \leq \lambda\left(T+T^{*}+\frac{V}{2 N}\right)^{\theta}-d T^{1+\theta}-\frac{\delta}{2}\left(T^{*}\right)^{1+\theta} \\
& -\frac{\delta}{4}\left(T^{*}\right)^{1+\theta}-\frac{c}{2}\left(\frac{V}{2 N}\right)^{1+\theta}+E \\
& \sigma^{2}\left[T^{1+\theta}+\left(T^{*}\right)^{1+\theta}+\left(\frac{V}{2 N}\right)^{1+\theta}\right] \leq-\frac{\theta}{2} T^{1+\theta} \\
&
\end{aligned}
$$

where

$$
\begin{aligned}
E & =\sup _{\left(T, T^{*}, V\right) \in \mathbb{R}_{+}^{3}}\left\{-\frac{1}{2}\left(d-\theta \sigma^{2}\right) T^{1+\theta}\right. \\
& -\frac{1}{4}\left(\delta-2 \theta \sigma^{2}\right)\left(T^{*}\right)^{1+\theta}-\frac{1}{2}\left(c-\theta \sigma^{2}\right)\left(\frac{V}{2 N}\right)^{1+\theta} \\
& \left.+\lambda\left(T+T^{*}+\frac{V}{2 N}\right)^{\theta}\right\}<\infty .
\end{aligned}
$$

Hence,

$$
\begin{aligned}
L \bar{W} \leq & -M \beta+(M+1) k V-\frac{\lambda}{T}-\frac{k T V}{T^{*}}-\frac{d}{2} T^{1+\theta} \\
& -\frac{\delta}{4}\left(T^{*}\right)^{1+\theta}-\frac{c}{2}\left(\frac{V}{2 N}\right)^{1+\theta}+E+d+\delta+\frac{1}{2} \sigma_{1}^{2} \\
& +\frac{1}{2} \sigma_{2}^{2} .
\end{aligned}
$$

Next, we construct a compact subset $D_{\varepsilon}$ such that condition (H2) in Lemma A.1 holds. Define the following bounded closed set:

$$
D_{\varepsilon}=\left\{\varepsilon \leq T \leq \frac{1}{\varepsilon}, \varepsilon^{3} \leq T^{*} \leq \frac{1}{\varepsilon^{3}}, \varepsilon \leq V \leq \frac{1}{\varepsilon}\right\},
$$

where $\varepsilon$ is a sufficiently small constant. In the set $\mathbb{R}_{+}^{3} \backslash D_{\varepsilon}$, we further choose $\varepsilon$ sufficiently small such that the following conditions hold:

$$
\begin{aligned}
-M \beta+(M+1) k \varepsilon+C & \leq-1, \\
-\frac{\lambda}{\varepsilon}+H & \leq-1, \\
-\frac{k}{\varepsilon}+H & \leq-1, \\
-\frac{c}{4(2 N \varepsilon)^{1+\theta}}+H & \leq-1, \\
-\frac{d}{4 \varepsilon^{1+\theta}}+H & \leq-1, \\
-\frac{\delta}{8 \varepsilon^{3(1+\theta)}}+H & \leq-1,
\end{aligned}
$$

where

$$
\begin{aligned}
H & =\sup _{\left(T, T^{*}, V\right) \in \mathbb{R}_{+}^{3}}\left\{(M+1) k V-\frac{d}{4} T^{1+\theta}-\frac{\delta}{8}\left(T^{*}\right)^{1+\theta}\right. \\
& \left.-\frac{c}{4}\left(\frac{V}{2 N}\right)^{1+\theta}+E+d+\delta+\frac{1}{2} \sigma_{1}^{2}+\frac{1}{2} \sigma_{2}^{2}\right\}<\infty .
\end{aligned}
$$

In the following, we separate $\mathbb{R}_{+}^{3} \backslash D_{\varepsilon}$ to six domains:

$$
\begin{aligned}
D_{1} & =\left\{\left(T, T^{*}, V\right) \in \mathbb{R}_{+}^{3}, 0<V<\varepsilon\right\}, \\
D_{2} & =\left\{\left(T, T^{*}, V\right) \in \mathbb{R}_{+}^{3}, 0<T<\varepsilon\right\}, \\
D_{3} & =\left\{\left(T, T^{*}, V\right) \in \mathbb{R}_{+}^{3}, 0<T^{*}<\varepsilon^{3}, T \geq \varepsilon, V \geq \varepsilon\right\}, \\
D_{4} & =\left\{\left(T, T^{*}, V\right) \in \mathbb{R}_{+}^{3}, \quad V>\frac{1}{\varepsilon}\right\}, \\
D_{5} & =\left\{\left(T, T^{*}, V\right) \in \mathbb{R}_{+}^{3}, T>\frac{1}{\varepsilon}\right\}, \\
D_{6} & =\left\{\left(T, T^{*}, V\right) \in \mathbb{R}_{+}^{3}, T^{*}>\frac{1}{\varepsilon^{3}}\right\} .
\end{aligned}
$$

Obviously, $D_{\varepsilon}^{c}=D_{1} \cup D_{2} \cup D_{3} \cup D_{4} \cup D_{5} \cup D_{6}$.

Case 1. If $\left(T, T^{*}, V\right) \in D_{1}$,

$$
\begin{aligned}
L \bar{W} \leq & -M \beta+(M+1) k V-\frac{d}{2} T^{1+\theta}-\frac{\delta}{4}\left(T^{*}\right)^{1+\theta} \\
& -\frac{c}{2}\left(\frac{V}{2 N}\right)^{1+\theta}+E+d+\delta+\frac{1}{2} \sigma_{1}^{2}+\frac{1}{2} \sigma_{2}^{2} \\
\leq & -M \beta+(M+1) k \varepsilon+C .
\end{aligned}
$$


According to (25), it implies that $L \bar{W} \leq-1$ for any $\left(T, T^{*}, V\right) \in D_{1}$.

Case 2. If $\left(T, T^{*}, V\right) \in D_{2}$,

$$
\begin{aligned}
L \bar{W} \leq & -\frac{\lambda}{T}+(M+1) k V-\frac{d}{2} T^{1+\theta}-\frac{\delta}{4}\left(T^{*}\right)^{1+\theta} \\
& -\frac{c}{2}\left(\frac{V}{2 N}\right)^{1+\theta}+E+d+\delta+\frac{1}{2} \sigma_{1}^{2}+\frac{1}{2} \sigma_{2}^{2} \\
\leq & -\frac{\lambda}{T}+H \leq-\frac{\lambda}{\varepsilon}+H .
\end{aligned}
$$

In view of (26), we have $L \bar{W} \leq-1$ for any $\left(T, T^{*}, V\right) \in D_{2}$.

Case 3. If $\left(T, T^{*}, V\right) \in D_{3}$,

$$
\begin{aligned}
L \bar{W} \leq & -\frac{k T V}{T^{*}}+(M+1) k V-\frac{d}{2} T^{1+\theta}-\frac{\delta}{4}\left(T^{*}\right)^{1+\theta} \\
& -\frac{c}{2}\left(\frac{V}{2 N}\right)^{1+\theta}+E+d+\delta+\frac{1}{2} \sigma_{1}^{2}+\frac{1}{2} \sigma_{2}^{2} \\
\leq & -\frac{k V T}{T^{*}}+H \leq-\frac{k}{\varepsilon}+H .
\end{aligned}
$$

According to (27), we deduce that $L \bar{W} \leq-1$ for any $\left(T, T^{*}, V\right) \in D_{3}$.

Case 4. If $\left(T, T^{*}, V\right) \in D_{4}$,

$$
\begin{aligned}
L \bar{W} \leq & -\frac{c}{2}\left(\frac{V}{2 N}\right)^{1+\theta}+(M+1) k V-\frac{d}{2} T^{1+\theta} \\
& -\frac{\delta}{4}\left(T^{*}\right)^{1+\theta}+E+d+\delta+\frac{1}{2} \sigma_{1}^{2}+\frac{1}{2} \sigma_{2}^{2} \\
\leq & -\frac{c}{4}\left(\frac{V}{2 N}\right)^{1+\theta}+H \leq-\frac{c}{4(2 N \varepsilon)^{1+\theta}}+H .
\end{aligned}
$$

In view of (30), we obtain that $L \bar{W} \leq-1$ for any $\left(T, T^{*}, V\right) \in$ $D_{4}$.

Case 5. If $\left(T, T^{*}, V\right) \in D_{4}$,

$$
\begin{aligned}
L \bar{W} \leq & -\frac{d}{2} T^{1+\theta}+(M+1) k V-\frac{\delta}{4}\left(T^{*}\right)^{1+\theta} \\
& -\frac{c}{2}\left(\frac{V}{2 N}\right)^{1+\theta}+E+d+\delta+\frac{1}{2} \sigma_{1}^{2}+\frac{1}{2} \sigma_{2}^{2} \\
\leq & -\frac{d}{4} T^{1+\theta}+H \leq-\frac{d}{4 \varepsilon^{1+\theta}}+H .
\end{aligned}
$$

By condition (28), we can conclude that $L \bar{W} \leq-1$ for all $\left(T, T^{*}, V\right) \in D_{5}$.

Case 6. If $\left(T, T^{*}, V\right) \in D_{5}$,

$$
\begin{aligned}
L \bar{W} \leq & -\frac{\delta}{4}\left(T^{*}\right)^{1+\theta}+(M+1) k V-\frac{d}{2} T^{1+\theta} \\
& -\frac{c}{2}\left(\frac{V}{2 N}\right)^{1+\theta}+E+d+\delta+\frac{1}{2} \sigma_{1}^{2}+\frac{1}{2} \sigma_{2}^{2} \\
\leq & -\frac{\delta}{8}\left(T^{*}\right)^{1+\theta}+H \leq-\frac{\delta}{8 \varepsilon^{3(1+\theta)}}+H .
\end{aligned}
$$

It follows that $L \bar{W} \leq-1$ for any $\left(T, T^{*}, V\right) \in D_{6}$ if the condition (29) is satisfied.

Obviously, from (33)-(38), there exists a sufficiently small $\varepsilon$, such that

$$
L \bar{W} \leq-1 \quad \forall\left(T, T^{*}, V\right) \in D_{\varepsilon}^{c} .
$$

According to Lemma A.1 in Appendix, we obtain that system (3) is ergodic and has a unique stationary distribution. This completes the proof.

Remark 3. Theorem 2 indicates that the virus prevails if the critical condition $R_{0, i}^{s}$ is greater than one. We should mention that the critical condition $R_{0, i}^{s}$ of the stochastic model (3) without white noises is consistent with the basic reproduction number $R_{0, i}$ of its corresponding deterministic model (1). This implies that the existence of the stationary distribution of our model is a generalization of its corresponding deterministic model to the stability of the chronic disease steady state.

\section{Extinction}

In this section, following the method in Theorem 3.1 of [30], we employ the comparison theorem of stochastic differential equation [31] and the law of large numbers [32] (see Appendix for details) to derive the sufficient conditions for extinction for the virus.

Theorem 4. Let $\left(T(t), T^{*}(t), V(t)\right)$ be the solution of system (3) with any initial value $\left(T(0), T^{*}(0), V(0)\right) \in \mathbb{R}_{+}^{3}$. If $d>$ $(1 / 2) \sigma_{1}^{2}$, then, for almost $\omega \in \Omega$, the solution $\left(T(t), T^{*}(t), V(t)\right)$ of system (3) satisfies

$$
\begin{array}{r}
\limsup _{t \rightarrow \infty} \frac{1}{t} \ln \left(\frac{N}{c+i k T_{0}} T^{*}(t)+\frac{\sqrt{R_{0, i}}}{c+i k T_{0}} V(t)\right) \leq m, \\
i=0 \text { or } 1, \text { a.s., }
\end{array}
$$

where $m=\min \left\{\delta, c+i k T_{0}\right\}\left(\sqrt{R_{0, i}}-1\right) \mathbf{I}_{\left\{R_{0, i} \leq 1\right\}}+\max \{\delta, c+$ $\left.i k T_{0}\right\}\left(\sqrt{R_{0, i}}-1\right) \mathbf{I}_{\left\{R_{0, i}>1\right\}}+i k T_{0}+k N T_{0} \sigma_{1}\left[R_{0, i}\left(2 d-\sigma_{1}^{2}\right)\right]^{-1 / 2}-$ $(1 / 2)\left(\sigma_{2}^{-2}+\sigma_{3}^{-2}\right)^{-1}$ and the distribution of $T(t)$ converges weakly to the measure which has the density given by

$$
\pi(x)=Q \sigma_{1}^{-2} x^{-\left(2+2 d / \sigma_{1}^{2}\right)} e^{-2 \lambda / \sigma_{1}^{2} x}, \quad x \in(0, \infty),
$$

where $Q=\sigma_{1}^{2}\left(2 \lambda / \sigma_{1}^{2}\right)^{1+2 d / \sigma_{1}^{2}} \Gamma^{-1}\left(2 d / \sigma_{1}^{2}+1\right)$ such that $\int_{0}^{\infty} \pi(x) d x=1$. If $m<0$, then the populations $T^{*}$ and $V$ will be eradicated with probability one; that is,

$$
\begin{gathered}
\lim _{t \rightarrow \infty} T^{*}(t)=0, \\
\lim _{t \rightarrow \infty} V(t)=0,
\end{gathered}
$$

a.s.

Proof. By Lemma 1, we know that the solution of system (3) is positive, and it is obvious that

$$
d T \leq(\lambda-d T) d t+\sigma_{1} T d B_{1}(t) .
$$


Consider the following auxiliary equation with stochastic differential equation:

$$
d x=(\lambda-d x) d t+\sigma_{1} x d B_{1}(t),
$$

with the initial value $x(0)=T(0)>0$. Corresponding to (A.4) in Appendix, this yields

$$
\begin{aligned}
& g(x)=\lambda-d x, \\
& \sigma(x)=\sigma_{1} x
\end{aligned}
$$

and we further calculate that

$$
x \in(0, \infty),
$$

$$
\begin{aligned}
\int_{\mathcal{C}_{1}}^{x} \frac{g(u)}{\sigma^{2}(u)} d u & =\frac{1}{\sigma_{1}^{2}} \int_{\mathcal{C}_{1}}^{x}\left(\frac{\lambda}{u^{2}}-\frac{d}{u}\right) d u \\
& =-\frac{1}{\sigma_{1}^{2}}\left[\frac{\lambda}{x}+d \ln x\right]+C_{1},
\end{aligned}
$$

where $c_{1}$ is an arbitrary fixed positive constant and $C_{1}$ is a constant determined by the above calculation. It is obvious that

$$
\begin{aligned}
& \int_{0}^{\infty} \frac{1}{\sigma^{2}(x)} \exp \left\{2 \int_{c_{1}}^{x} \frac{g(u)}{\sigma^{2}(u)} d u\right\} d x \\
& \quad=Q \sigma_{1}^{-2} \int_{0}^{\infty} x^{-\left(2+2 d / \sigma_{1}^{2}\right)} e^{-2 \lambda / \sigma_{1}^{2} x} d x<\infty,
\end{aligned}
$$

where $Q=e^{2 C_{1}}$. By Lemma A.2 in Appendix, we obtain that system (44) has the ergodic property with ergodic distribution:

$$
\pi(x)=Q \sigma_{1}^{-2} x^{-\left(2+2 d / \sigma_{1}^{2}\right)} e^{-2 \lambda / \sigma_{1}^{2} x}, \quad x \in(0, \infty),
$$

where $Q=\sigma_{1}^{2}\left(2 \lambda / \sigma_{1}^{2}\right)^{1+2 d / \sigma_{1}^{2}} \Gamma^{-1}\left(2 d / \sigma_{1}^{2}+1\right)$ such that $\int_{0}^{\infty} \pi(x) d x=1$. It then follows that

$$
\lim _{t \rightarrow \infty} \frac{1}{t} \int_{0}^{t} x(s) d s=\int_{0}^{\infty} x \pi(x) d x \quad \text { a.s. }
$$

Let $x(t)$ be the solution of system (44) with the initial value $x(0)=T(0)>0$, by the comparison theorem of stochastic differential equation [31], we obtain that

$$
T(t) \leq x(t) \quad \text { a.s. }
$$

We further calculate that

$$
\begin{aligned}
A_{1} & :=\int_{0}^{\infty} x \pi(x) d x \\
& =Q \sigma_{1}^{-2} \int_{0}^{\infty} x^{-\left(1+2 d / \sigma_{1}^{2}\right)} e^{-2 \lambda / \sigma_{1}^{2} x} d x \\
& =Q \sigma_{1}^{-2}\left(\frac{2 \lambda}{\sigma_{1}^{2}}\right)^{-2 d / \sigma_{1}^{2}} \int_{0}^{\infty} u^{2 d / \sigma_{1}^{2}-1} e^{-u} d u \\
& =Q \sigma_{1}^{-2}\left(\frac{2 \lambda}{\sigma_{1}^{2}}\right)^{-2 d / \sigma_{1}^{2}} \Gamma\left(\frac{2 d}{\sigma_{1}^{2}}\right)=\frac{2 \lambda}{\sigma_{1}^{2}} \frac{\Gamma\left(2 d / \sigma_{1}^{2}\right)}{\Gamma\left(2 d / \sigma_{1}^{2}+1\right)} \\
& =\frac{\lambda}{d},
\end{aligned}
$$

$$
\begin{aligned}
A_{2} & :=\int_{0}^{\infty} x^{2} \pi(x) d x=Q \sigma_{1}^{-2} \int_{0}^{\infty} x^{-2 d / \sigma_{1}^{2}} e^{-2 \lambda / \sigma_{1}^{2} x} d x \\
& =Q \sigma_{1}^{-2}\left(\frac{2 \lambda}{\sigma_{1}^{2}}\right)^{\left(1-2 d / \sigma_{1}^{2}\right)} \int_{0}^{\infty} u^{2 d / \sigma_{1}^{2}-2} e^{-u} d u \\
& =Q \sigma_{1}^{-2}\left(\frac{2 \lambda}{\sigma_{1}^{2}}\right)^{\left(1-2 d / \sigma_{1}^{2}\right)} \Gamma\left(\frac{2 d}{\sigma_{1}^{2}}-1\right) \\
& =\left(\frac{2 \lambda}{\sigma_{1}^{2}}\right)^{2} \frac{\Gamma\left(2 d / \sigma_{1}^{2}-1\right)}{\Gamma\left(2 d / \sigma_{1}^{2}+1\right)}=\frac{2 \lambda^{2}}{d\left(2 d-\sigma_{1}^{2}\right)} .
\end{aligned}
$$

Therefore,

$$
\begin{aligned}
\int_{0}^{\infty} & \left(x-T_{0}\right)^{2} \pi(x) d x \\
\quad= & \int_{0}^{\infty} x^{2} \pi(x) d x-T_{0} \int_{0}^{\infty} x \pi(x) d x+T_{0}^{2} \\
= & A_{2}-2 T_{0} A_{1}+T_{0}^{2} \\
= & \frac{2 \lambda^{2}}{d\left(2 d-\sigma_{1}^{2}\right)}-2\left(\frac{\lambda}{d}\right)^{2}+\left(\frac{\lambda}{d}\right)^{2}=\frac{\lambda^{2} \sigma_{1}^{2}}{d^{2}\left(2 d-\sigma_{1}^{2}\right)} .
\end{aligned}
$$

Denote the matrix

$$
F=\left[\begin{array}{cc}
0 & \frac{k T_{0}}{\delta} \\
\frac{N \delta}{c+i k T_{0}} & 0
\end{array}\right] .
$$

By Theorem 1.4 of literature [33], we know that there must be a left eigenvector $\left(\omega_{1}, \omega_{2}\right)$ of matrix $F$ corresponding to the value $\sqrt{R_{0, i}}$, such that

$$
\left(\omega_{1}, \omega_{2}\right) F=\sqrt{R_{0, i}}\left(\omega_{1}, \omega_{2}\right)
$$

where $\left(\omega_{1}, \omega_{2}\right)=\left(N \delta /\left(c+i k T_{0}\right), \sqrt{R_{0, i}}\right)$.

Define a $C^{2}$-function $W: \mathbb{R}_{+}^{3} \rightarrow \mathbb{R}$ :

$$
G\left(T^{*}, V\right)=\alpha_{1} T^{*}+\alpha_{2} V
$$

where $\alpha_{1}=\omega_{1} / \delta, \alpha_{2}=\omega_{2} /\left(c+i k T_{0}\right)$. Applying Itô's formula yields

$$
\begin{aligned}
d(\ln G)= & L(\ln G) d t \\
& +\frac{1}{G}\left(\alpha_{1} \sigma_{2} T^{*} d B_{2}(t)+\alpha_{2} \sigma_{3} V d B_{3}(t)\right),
\end{aligned}
$$

where

$$
\begin{aligned}
L(\ln G)= & \frac{\alpha_{1}}{G}\left(k T V-\delta T^{*}\right) \\
& +\frac{\alpha_{2}}{G}\left(N \delta T^{*}-c V-i k T V\right)-\frac{\alpha_{1}^{2} \sigma_{2}^{2}\left(T^{*}\right)^{2}}{2 G^{2}} \\
& -\frac{\alpha_{2}^{2} \sigma_{3}^{2} V^{2}}{2 G^{2}}:=B-\frac{\alpha_{1}^{2} \sigma_{2}^{2}\left(T^{*}\right)^{2}}{2 G^{2}}-\frac{\alpha_{2}^{2} \sigma_{3}^{2} V^{2}}{2 G^{2}} .
\end{aligned}
$$


Furthermore, we have

$$
\begin{aligned}
& G^{2}=\left(\alpha_{1} \sigma_{2} T^{*} \frac{1}{\sigma_{2}}+\alpha_{2} \sigma_{3} V \frac{1}{\sigma_{3}}\right)^{2} \leq\left[\alpha_{1}^{2} \sigma_{2}^{2}\left(T^{*}\right)^{2}\right. \\
& \left.+\alpha_{2}^{2} \sigma_{3}^{2} V^{2}\right]\left(\frac{1}{\sigma_{2}^{2}}+\frac{1}{\sigma_{3}^{2}}\right) \\
& B=\frac{1}{G}\left[\alpha_{1}\left(k T V-\delta T^{*}\right)+\alpha_{2}\left(N \delta T^{*}-c V-i k T V\right)\right] \\
& =\frac{1}{G}\left\{\alpha_{1}\left[k\left(T-T_{0}\right) V+k T_{0} V-\delta T^{*}\right]\right. \\
& \left.+\alpha_{2}\left[N \delta T^{*}-c V-i k\left(T-T_{0}\right) V-i k T_{0} V\right]\right\} \\
& =\frac{\alpha_{1} k V}{G}\left(T-T_{0}\right)+\frac{1}{G}\left[\alpha_{1}\left(k T_{0} V-\delta T^{*}\right)\right. \\
& \left.+\alpha_{2}\left(N \delta T^{*}-c V-i k T_{0} V\right)\right]-\frac{\alpha_{2}}{G} i k\left(T-T_{0}\right) V \\
& \leq \frac{\alpha_{1} k}{\alpha_{2}}\left|T-T_{0}\right|+\frac{1}{G}\left[\frac{\omega_{1}}{\delta}\left(k T_{0} V-\delta T^{*}\right)\right. \\
& \left.+\frac{\omega_{2}}{c+i k T_{0}}\left(N \delta T^{*}-c V-i k T_{0} V\right)\right]+i k T_{0} \\
& =\frac{\alpha_{1} k}{\alpha_{2}}\left|T-T_{0}\right|+\frac{1}{G}\left(\omega_{1}, \omega_{2}\right)\left[F\left(T^{*}, V\right)^{T}\right. \\
& \left.-\left(T^{*}, V\right)^{T}\right]+i k T_{0}=\frac{\alpha_{1} k}{\alpha_{2}}\left|T-T_{0}\right|+i k T_{0} \\
& +\frac{1}{G}\left(\sqrt{R_{0, i}}-1\right)\left(\omega_{1} T^{*}+\omega_{2} V\right)=\frac{\alpha_{1} k}{\alpha_{2}}\left|T-T_{0}\right| \\
& +i k T_{0}+\frac{1}{G}\left(\sqrt{R_{0, i}}-1\right)\left(\alpha_{1} \delta T^{*}+\left(c+i k T_{0}\right) \alpha_{2} V\right) \\
& \leq \frac{\alpha_{1} k}{\alpha_{2}}\left|T-T_{0}\right|+i k T_{0}+\min \left\{\delta, c+i k T_{0}\right\}\left(\sqrt{R_{0, i}}\right. \\
& \text { - 1) } \mathbf{I}_{\left\{R_{0, i} \leq 1\right\}}+\max \left\{\delta, c+i k T_{0}\right\}\left(\sqrt{R_{0, i}}-1\right) \\
& \text { - } \mathbf{I}_{\left\{R_{0, i}>1\right\}} \text {. }
\end{aligned}
$$

According to (58), we get that

$$
\begin{aligned}
L(\ln G) \leq & \min \left\{\delta, c+i k T_{0}\right\}\left(\sqrt{R_{0, i}}-1\right) \mathbf{I}_{\left\{R_{0, i} \leq 1\right\}} \\
& +\max \left\{\delta, c+i k T_{0}\right\}\left(\sqrt{R_{0, i}}-1\right) \mathbf{I}_{\left\{R_{0, i}>1\right\}} \\
& +\frac{\alpha_{1} k}{\alpha_{2}}\left|T-T_{0}\right|+i k T_{0}-\frac{1}{2}\left(\sigma_{2}^{-2}+\sigma_{3}^{-2}\right)^{-1} .
\end{aligned}
$$

From (56), we further obtain that

$$
\begin{aligned}
& d(\ln G) \leq\left[\min \left\{\delta, c+i k T_{0}\right\}\left(\sqrt{R_{0, i}}-1\right) \mathbf{I}_{\left\{R_{0, i} \leq 1\right\}}\right. \\
& +\max \left\{\delta, c+i k T_{0}\right\} \times\left(\sqrt{R_{0, i}}-1\right) \mathbf{I}_{\left\{R_{0, i}>1\right\}}
\end{aligned}
$$

$$
\begin{aligned}
& \left.+\frac{\alpha_{1} k}{\alpha_{2}}\left|T-T_{0}\right|+i k T_{0}-\frac{1}{2}\left(\sigma_{2}^{-2}+\sigma_{3}^{-2}\right)^{-1}\right] d t \\
& +\frac{1}{G}\left(\alpha_{1} \sigma_{2} T^{*} d B_{2}(t)+\alpha_{2} \sigma_{3} V d B_{3}(t)\right) .
\end{aligned}
$$

Integrating (60) from 0 to $t$, then dividing by $t$ on both sides, yields

$$
\begin{aligned}
& \frac{\ln G(t)}{t} \leq \frac{\ln G(0)}{t} \\
& +\min \left\{\delta, c+i k T_{0}\right\}\left(\sqrt{R_{0, i}}-1\right) \mathbf{I}_{\left\{R_{0, i} \leq 1\right\}} \\
& +\max \left\{\delta, c+i k T_{0}\right\} \times\left(\sqrt{R_{0, i}}-1\right) \mathbf{I}_{\left\{R_{0, i}>1\right\}} \\
& +\frac{\alpha_{1} k}{\alpha_{2} t} \int_{0}^{t}\left|T(s)-T_{0}\right| d s+i k T_{0} \\
& -\frac{1}{2}\left(\sigma_{2}^{-2}+\sigma_{3}^{-2}\right)^{-1} \\
& +\frac{1}{t} \int_{0}^{t} \frac{\alpha_{1} \sigma_{2} T^{*}(s)}{G(s)} d B_{2}(s) \\
& +\frac{1}{t} \int_{0}^{t} \frac{\alpha_{2} \sigma_{3} V(s)}{G(s)} d B_{3}(s) \\
& =\frac{\ln G(0)}{t} \\
& +\min \left\{\delta, c+i k T_{0}\right\}\left(\sqrt{R_{0, i}}-1\right) \mathbf{I}_{\left\{R_{0, i} \leq 1\right\}} \\
& +\max \left\{\delta, c+i k T_{0}\right\} \times\left(\sqrt{R_{0, i}}-1\right) \mathbf{I}_{\left\{R_{0, i}>1\right\}} \\
& +\frac{\alpha_{1} k}{\alpha_{2} t} \int_{0}^{t}\left|T(s)-T_{0}\right| d s+i k T_{0} \\
& -\frac{1}{2}\left(\sigma_{2}^{-2}+\sigma_{3}^{-2}\right)^{-1}+\frac{M_{1}(t)}{t}+\frac{M_{2}(t)}{t},
\end{aligned}
$$

where

$$
\begin{aligned}
& M_{1}(t)=\int_{0}^{t} \frac{\alpha_{1} \sigma_{2} T^{*}(s)}{G(s)} d B_{2}(s), \\
& M_{2}(t)=\int_{0}^{t} \frac{\alpha_{2} \sigma_{3} V(s)}{G(s)} d B_{3}(s) .
\end{aligned}
$$

$M_{1}(t)$ and $M_{2}(t)$ are local martingales whose quadratic variations are $\left\langle M_{1}, M_{1}\right\rangle_{t}=\sigma_{2}^{2} \times \int_{0}^{t}\left(\alpha_{1} T^{*}(s) / G(s)\right) d s \leq \sigma_{2}^{2} t$ and $\left\langle M_{2}, M_{2}\right\rangle_{t}=\sigma_{3}^{2} \int_{0}^{t}\left(\alpha_{2} V(s) / G(s)\right) d s \leq \sigma_{3}^{2} t$. Applying the strong law of large numbers for local martingale [29] yields

$$
\lim _{t \rightarrow \infty} \frac{M_{i}(t)}{t}=0 \quad \text { a.s., } i=1,2 .
$$

Since $T(t)$ is ergodic and $\int_{0}^{\infty} x \pi(x) d x<\infty$, so

$$
\lim _{t \rightarrow \infty} \frac{1}{t} \int_{0}^{t}\left|T(s)-T_{0}\right| d s=\int_{0}^{\infty}\left|x-T_{0}\right| \pi(x) d x
$$




$$
\begin{aligned}
& \leq\left(\int_{0}^{\infty}\left(x-T_{0}\right)^{2} \pi(x) d x\right)^{1 / 2} \\
& =T_{0} \sigma_{1}\left(2 d-\sigma_{1}^{2}\right)^{-1 / 2}
\end{aligned}
$$

Taking the superior limit on both sides of inequality (61) and combining with (63) and (64), we obtain

$$
\begin{aligned}
\limsup _{t \rightarrow \infty} & \frac{\ln G(t)}{t} \\
\leq & \min \left\{\delta, c+i k T_{0}\right\}\left(\sqrt{R_{0, i}}-1\right) \mathbf{I}_{\left\{R_{0, i} \leq 1\right\}} \\
& +\max \left\{\delta, c+i k T_{0}\right\} \times\left(\sqrt{R_{0, i}}-1\right) \mathbf{I}_{\left\{R_{0, i}>1\right\}} \\
& +\frac{\alpha_{1} k}{\alpha_{2} t} \int_{0}^{t}\left|T(s)-T_{0}\right| d s+i k T_{0} \\
& -\frac{1}{2}\left(\sigma_{2}^{-2}+\sigma_{3}^{-2}\right)^{-1} \\
\leq & \min \left\{\delta, c+i k T_{0}\right\}\left(\sqrt{R_{0, i}}-1\right) \mathbf{I}_{\left\{R_{0, i} \leq 1\right\}} \\
& +\max \left\{\delta, c+i k T_{0}\right\} \times\left(\sqrt{R_{0, i}}-1\right) \mathbf{I}_{\left\{R_{0, i}>1\right\}} \\
& +i k T_{0}+k N T_{0} \sigma_{1}\left[R_{0, i}\left(2 d-\sigma_{1}^{2}\right)\right]^{-1 / 2} \\
& -\frac{1}{2}\left(\sigma_{2}^{-2}+\sigma_{3}^{-2}\right)^{-1}:=m \text { a.s., }
\end{aligned}
$$

which is the required statement. For the special case, if $m<0$, then it is concluded that

$$
\begin{aligned}
& \lim _{t \rightarrow \infty} \frac{\ln T^{*}(t)}{t}=0, \\
& \lim _{t \rightarrow \infty} \frac{\ln V(t)}{t}=0
\end{aligned}
$$

a.s.,

which means that $\lim _{t \rightarrow \infty} T^{*}(t)=0$ and $\lim _{t \rightarrow \infty} V(t)=0$ a.s. In other words, the populations $T^{*}$ and $V$ will tend to zero exponentially with probability one. This completes the proof.

\section{Numerical Simulations}

In Sections 3 and 4, we obtained the stationary distribution and extinction of the virus under certain conditions, respectively. We now explore the cell and viral dynamics under different white noises. Here, by employing the Milstein's higher order method in Higham [34], the discrete form of model (3) is

$$
\begin{aligned}
T_{j+1}= & T_{j}+\left(\lambda-d T_{j}-k T_{j} V_{j}\right) \Delta t+\sigma_{1} T_{j} \sqrt{\Delta t} \xi_{j} \\
& +\frac{\sigma_{1}^{2}}{2} T_{j}\left(\Delta t \xi_{j}^{2}-\Delta t\right), \\
T_{j+1}^{*}= & T_{j}^{*}+\left(k T_{j} V_{j}-\delta T_{j}^{*}\right) \Delta t+\sigma_{2} T_{j}^{*} \sqrt{\Delta t} \eta_{j} \\
& +\frac{\sigma_{2}^{2}}{2} T_{j}^{*}\left(\Delta t \eta_{j}^{2}-\Delta t\right),
\end{aligned}
$$

$$
\begin{aligned}
V_{j+1}= & V_{j}+\left(N \delta T_{j}^{*}-c V_{j}-i k T_{j} V_{j}\right) \Delta t+\sigma_{3} V_{j} \sqrt{\Delta t} \phi_{j} \\
& +\frac{\sigma_{3}^{2}}{2} V_{j}\left(\Delta t \phi_{j}^{2}-\Delta t\right), \quad i=0 \text { or } 1
\end{aligned}
$$

where $\xi_{j}, \eta_{j}$, and $\phi_{j}, j=1,2, \ldots, n$, are the $j$ th realization of three independent Gaussian random variables with distribution $N(0,1)$, and the time increment $\Delta t=0.015$.

In the following, for the sake of representativeness, we take $i=1$ for system (3) as an example, and we can carry out same simulations as follows when $i=0$. The parameters are chosen from the actual parameter values of previous references (see Table 1), and we calculate that the basic reproduction number $R_{0,1}=5.2609>1$; from Section 2, we know that the chronic disease steady state is globally asymptotically stable. In order to examine how white noises affect model dynamic behavior, we choose three pairs of white noise set $\left(\sigma_{1}, \sigma_{2}, \sigma_{3}\right)$ equal to $(0.008,0.3,1)$, $(0.01,0.4,1.2)$, and $(0.02,0.8,1.5)$, with the critical condition $R_{0,1}^{s}$ equal to $3.0853,2.6928$, and 1.5107 , respectively. For these parameter sets, Theorem 2 is satisfied and system (3) has a unique stationary distribution. By simulations, for each pair of white noise sets chosen above, Figures 1(a) and 2(a) demonstrate the solutions of the stochastic model (3) and the corresponding deterministic model (1) initiated at the initial value $\left(T(0), T^{*}(0), V(0)\right)=(1000,0.001,0.001)$, and Figures 1(b) and 2(b) show histograms of the approximate stationary distributions for two components ( $T$ and $V$ ) from 10000 simulation runs at time $t=300$. The figures indicate that the small magnitudes of random fluctuations can generate the irregular recurrence of populations $T$ and $V$.

Using the data values in Table 1, we further choose two pairs of white noise set $\left(\sigma_{1}, \sigma_{2}, \sigma_{3}\right)$ equal to $(0.03,1.2,2.5)$ and $(0.06,1.8,3.5)$, with the critical condition $R_{0,1}^{s}$ equal to 0.7850 and 0.2584 , respectively. We do not give theoretical analysis in these cases, and now we take numerical simulation to discuss the effect of random fluctuations on model behavior. Figure 3 shows that, as the white noise set is increasing, the virus can be eradicated for stochastic model (3), while the virus is still persistent for its corresponding deterministic model (1). It means that the large magnitudes of random fluctuations could lead to the extinction of the virus.

\section{Conclusions}

We have studied a stochastic virus infection model with or without the virus loss term. Mathematically, we have given a systematic method to the analysis (stationary distribution and extinction) of such three-dimensional stochastic viral dynamic model. If the critical condition $R_{0, i}^{s}(i=0$ or 1$)$ is greater than one, then system (3) has a unique stationary distribution. Furthermore, we obtain the sufficient conditions to ensure the extinction of the virus by employing the comparison theorem of stochastic differential equation and the law of large numbers. Numerical simulations demonstrate that, on one hand, healthy target cells and virions can be maintained within a certain range for the small magnitudes 
TABLE 1: List of parameters.

\begin{tabular}{lcccc}
\hline Parameters & Description & Unit & Value & Source \\
\hline$\lambda$ & Target cells source term & $\mu \mathrm{l}^{-1}$ day $^{-1}$ & 10 & {$[5,6,9]$} \\
$d$ & Death rate of healthy target cells & Day $^{-1}$ & 0.007 & {$[5,6]$} \\
$k$ & Viral infectivity rate & $\mu$ day $^{-1}$ & 0.000024 & {$[5,6,9]$} \\
$\delta$ & Death rate of infected target cells & Day $^{-1}$ & 1 & 2000 \\
$N$ & Burst term & Virions/cell & {$[5]$} \\
$c$ & Clearance rate of virus & Day $^{-1}$ & {$[5,6,9]$} \\
\hline
\end{tabular}
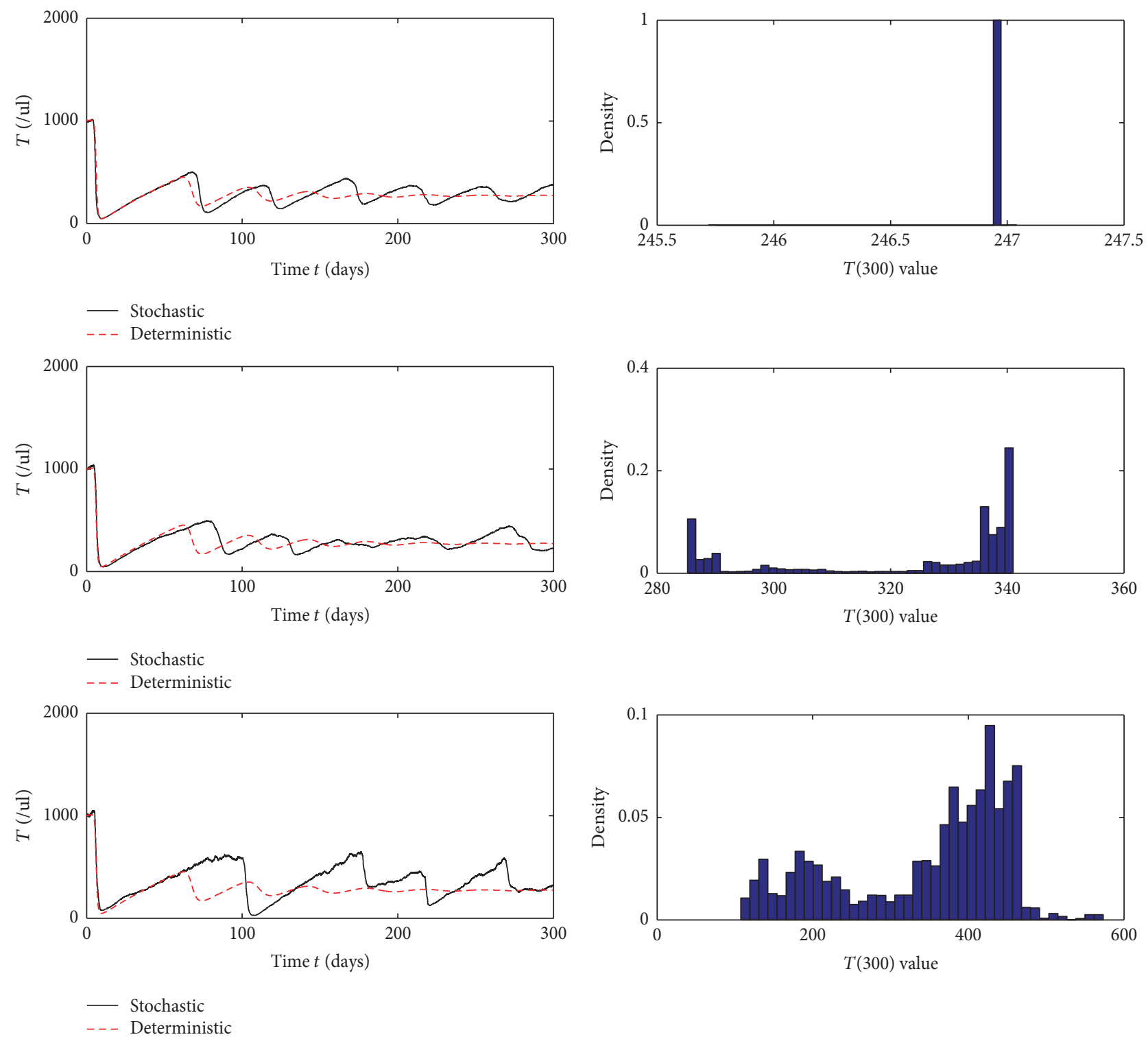

(a)

(b)

FIGURE 1: (a) Simulations of healthy target cells for the stochastic model (3) and its corresponding deterministic model (1) with three different sets of white noise. (b) Probability density of the values of the path $T(t)$ at $t=300$ for system (3) based on 10000 simulation runs. For parameters see Table 1 with $\left(\sigma_{1}, \sigma_{2}, \sigma_{3}\right)$ equal to $(0.008,0.3,1),(0.01,0.4,1.2)$, and $(0.02,0.8,1.5)$, respectively. 

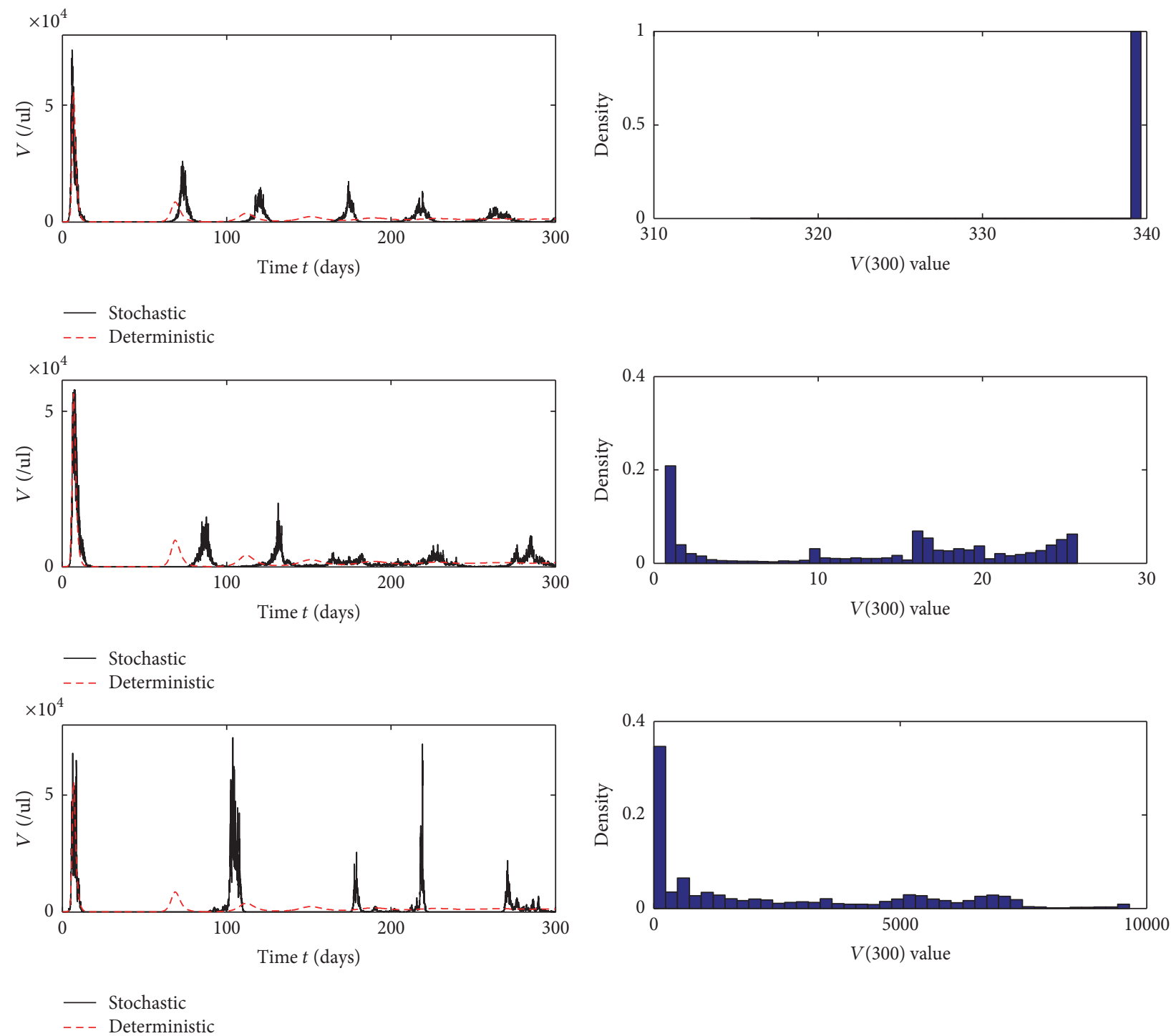

(a)

(b)

Figure 2: (a) Simulations of virus for the stochastic model (3) and its corresponding deterministic model (1) with three different sets of white noise. (b) Probability density of the values of the path $V(t)$ at $t=300$ for system (3) based on 10000 simulation runs. For parameters see Table 1 with $\left(\sigma_{1}, \sigma_{2}, \sigma_{3}\right)$ equal to $(0.008,0.3,1),(0.01,0.4,1.2)$, and $(0.02,0.8,1.5)$, respectively.

of white noises, which means that the virus is persistent; on the other hand, the virus could be cleared out for large magnitudes of white noises, which indicates the extinction of the virus.

In fact, the theoretical analysis results of system (3) can also be extended to the following viral infection model with combination drug therapy:

$$
\begin{aligned}
d T= & {\left[\lambda-d T-k\left(1-n_{r t}\right) T V\right] d t+\sigma_{1} T d B_{1}(t), } \\
d T^{*}= & {\left[k\left(1-n_{r t}\right) T V-\delta T^{*}\right] d t+\sigma_{2} T^{*} d B_{2}(t), } \\
d V= & {\left[N\left(1-n_{p}\right) \delta T^{*}-c V-i k T V\right] d t } \\
& +\sigma_{3} V d B_{3}(t), \quad i=0 \text { or } 1,
\end{aligned}
$$

where $n_{r t}$ and $n_{p}$ are the drug efficacies of different types of drugs. Therefore, our model as well as the theoretical analysis results are extension of the literature [18].

We have theoretically analyzed the existence of the stationary distribution and extinction for a stochastic HIV infection model with logistic T-cell and CTL immune response in literature [23]. Thus, with the work of this article, we may perform a similar study for our model in literature [5] with white noises:

$$
\begin{aligned}
& d T \\
& =\left[\lambda-d T+r T\left(1-\frac{T+T^{*}}{T_{\max }}\right)-k\left(1-n_{r t}\right) V T\right] d t \\
& +\sigma_{1} T d B_{1}(t),
\end{aligned}
$$



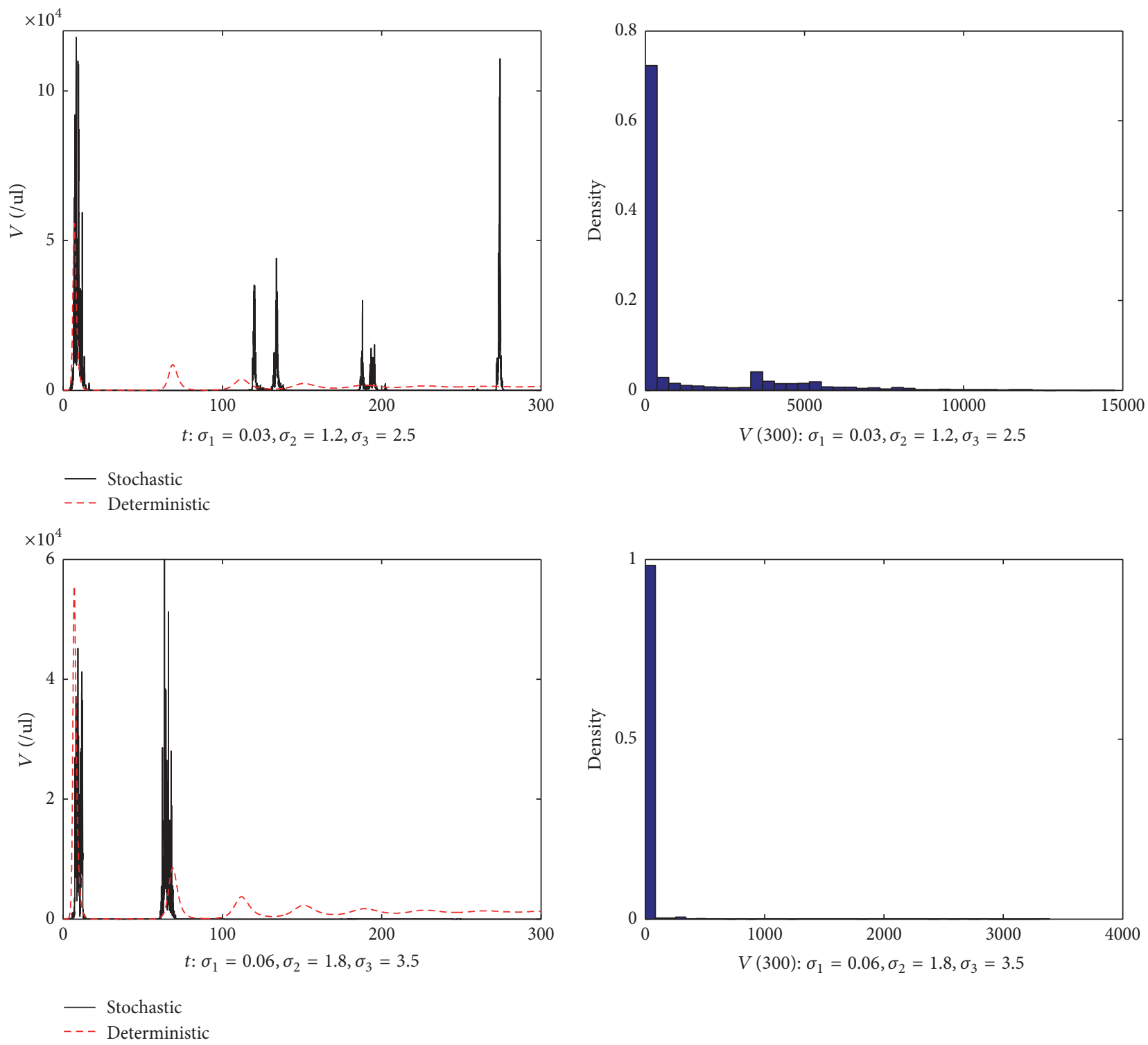

FIgURE 3: The paths of $V(t)$ for stochastic model (3) and the solutions of $V(t)$ for its corresponding deterministic model $(1)$ with $\left(\sigma_{1}, \sigma_{2}, \sigma_{3}\right)$ equal to $(0.03,1.2,2.5)$ and $(0.06,1.8,3.5)$, respectively.

$$
\begin{aligned}
& d T^{*} \\
& =\left[k\left(1-n_{r t}\right) V T-\delta T^{*}-q T^{*} Z\right] d t \\
& \quad+\sigma_{2} T^{*} d B_{2}(t), \\
& d V \\
& =\left[N \delta\left(1-n_{p}\right) T^{*}-(c+i k T) V\right] d t+\sigma_{3} V d B_{3}(t), \\
& d Z=\left(p T^{*} Z-d_{Z} Z\right) d t+\sigma_{4} Z d B_{4}(t),
\end{aligned}
$$

where $Z$ denotes the cytotoxic T lymphocyte (CTL) immune response, $q$ is the CTL activation rate, $p$ is the CTL responsiveness, and $d_{Z}$ is the death rate of CTL. $B_{4}(t)$ is independent standard Brownian motion with $B_{4}(0)=0$ and $\sigma_{4}^{2}>0$ denote the intensity of the white noise. All the other parameters are the same as those in systems (1), (3), and (68). This kind of model will be our future work.

\section{Appendix}

In this section, we introduce the theory of Hasminskii [28], which has been applied to show the existence of stationary distribution in Section 3.

Let $X(t)$ be usual time-homogeneous Markov process in $\mathbb{R}^{l}$ described by the following stochastic differential equation:

$$
d X(t)=b(X) d t+\sum_{r=1}^{k} g_{r}(X) d B_{r}(t)
$$


The diffusion matrix is defined as follows:

$$
A(x)=\left(a_{i j}(x)\right), \quad a_{i j}(x)=\sum_{r=1}^{k} g_{r}^{i}(x) g_{r}^{j}(x),
$$

where $X(t)$ is nonsingular.

Lemma A.1 (see chapter 4 of [28]). If there exists a bounded open domain $U \subset \mathbb{R}^{l}$ with regular boundary $\Gamma$, having properties

(H1) there exists a positive number $E$ such that $\sum_{i, j=1}^{l} a_{i j}(x) \xi_{i} \xi_{i} \geq E|\xi|^{2}$, for $x \in U$ and $\xi \in \mathbb{R}^{l}$,

(H2) there exists a nonnegative $C^{2}$-function $W$ such that $L W$ is negative for any $\mathbb{R}^{l} \backslash D$, then

$$
\mathbb{P}\left\{\lim _{t \rightarrow \infty} \frac{1}{t} \int_{0}^{t} f(X(s)) d s=\int_{\mathbb{R}}^{l} f(x) \mu(d x)\right\}=1
$$

for all $x \in \mathbb{R}^{l}$, where $\mu$ is the stationary distribution of the process $X(t)$, and $f(x)$ is a function integrable with respect to the measure $\mu$. Then, the Markov process $X(t)$ has a unique ergodic stationary distribution $\mu(\cdot)$.

Next, we introduce the law of large numbers theorem [32], which has been used to prove the extinction of system (3) in Section 4.

Consider the following one-dimensional homogeneous Markov process:

$$
\begin{aligned}
& d x(t)=g(x(t)) d t+\sigma(x(t)) d B(t) \\
& x\left(t_{0}\right)=x_{0}
\end{aligned}
$$

$$
t \geq t_{0}
$$

where $g(x)$ and $\sigma(x)$ are measurable functions from $\mathbb{R}$ to $\mathbb{R}$, and $B(t)$ is the Brownian movement. It is assumed that the functions $g(x), \sigma(x)$, and $1 / \sigma(x)$ are all locally bounded.

(H3) the functions $g(x)$ and $\sigma(x)$ are such that

$$
\begin{gathered}
Q(x)=\int_{0}^{x} \exp \left\{-2 \int_{0}^{s} \frac{g(u)}{\sigma^{2}(u)} d u\right\} d s \longrightarrow \pm \infty, \\
\text { as } x \longrightarrow \pm \infty, \\
R=\int_{-\infty}^{\infty} \frac{1}{\sigma^{2}(x)} \exp \left\{2 \int_{0}^{x} \frac{g(u)}{\sigma^{2}(u)} d u\right\} d x<\infty .
\end{gathered}
$$

Lemma A.2 (see theorem 1.16 of [32]). If (H3) is satisfied, then the stochastic process (A.4) has ergodic properties with the density given by

$$
\pi(x)=\frac{1}{R \sigma^{2}(x)} \exp \left\{2 \int_{0}^{x} \frac{g(u)}{\sigma^{2}(u)} d u\right\} .
$$

\section{Conflicts of Interest}

The authors declare that there are no conflicts of interest regarding the publication of this paper.

\section{Acknowledgments}

Y. Wang is supported by National Natural Science Foundation of China (no. 11301543, no. 11401589) and the Fundamental Research Funds for the Central Universities (no. 17CX02066). D. Jiang is supported by National Natural Science Foundation of China (no. 11371085) and the Fundamental Research Funds for the Central Universities (no. 15CX08011A).

\section{References}

[1] M. A. Nowak, S. Bonhoeffer, A. M. Hill, R. Boehme, H. C. Thomas, and H. Mcdade, "Viral dynamics in hepatitis B virus infection," Proceedings of the National Acadamy of Sciences of the United States of America, vol. 93, no. 9, pp. 4398-4402, 1996.

[2] M. A. Nowak and R. M. May, Virus Dynamics: Mathematics Principles of Immunology and Virology, Oxford University Press, London, UK, 2000.

[3] A. S. Perelson and P. W. Nelson, "Mathematical analysis of HIV1 dynamics in vivo," SIAM Review, vol. 41, no. 1, pp. 3-44, 1999.

[4] P. de Leenheer and H. L. Smith, "Virus dynamics: a global analysis," SIAM Journal on Applied Mathematics, vol. 63, no. 4, pp. 1313-1327, 2003.

[5] Y. Wang, Y. Zhou, F. Brauer, and J. M. Heffernan, "Viral dynamics model with CTL immune response incorporating antiretroviral therapy," Journal of Mathematical Biology, vol. 67, no. 4, pp. 901-934, 2013.

[6] Y. Wang, Y. Zhou, J. Wu, and J. Heffernan, "Oscillatory viral dynamics in a delayed HIV pathogenesis model," Mathematical Biosciences, vol. 219, no. 2, pp. 104-112, 2009.

[7] G. Huang, Y. Takeuchi, and W. Ma, "Lyapunov functionals for delay differential equations model of viral infections," SIAM Journal on Applied Mathematics, vol. 70, no. 7, pp. 2693-2708, 2010.

[8] M. Y. Li and H. Shu, "Impact of intracellular delays and targetcell dynamics on in vivo viral infections," SIAM Journal on Applied Mathematics, vol. 70, no. 7, pp. 2434-2448, 2010.

[9] R. V. Culshaw and S. Ruan, "A delay-differential equation model of HIV infection of $\mathrm{CD}^{+}{ }^{+}$T-cells," Mathematical Biosciences, vol. 165, no. 1, pp. 27-39, 2000.

[10] K. Hattaf and N. Yousfi, "Global stability of a virus dynamics model with cure rate and absorption," Journal of the Egyptian Mathematical Society, 2014.

[11] A. Singh, B. Razooky, C. D. Cox, M. L. Simpson, and L. S. Weinberger, "Transcriptional bursting from the HIV-1 promoter is a significant source of stochastic noise in HIV-1 gene expression," Biophysical Journal, vol. 98, no. 8, pp. L32-L34, 2010.

[12] X. Mao, G. Marion, and E. Renshaw, "Environmental Brownian noise suppresses explosions in population dynamics," Stochastic Processes and Their Applications, vol. 97, no. 1, pp. 95-110, 2002.

[13] Y. Liang, D. Greenhalgh, and X. Mao, "A stochastic differential equation model for the spread of HIV amongst people who inject drugs," Computational and Mathematical Methods in Medicine, vol. 2016, Article ID 6757928, 14 pages, 2016.

[14] D. Greenhalgh, Y. Liang, and X. Mao, "Modelling the effect of telegraph noise in the SIRS epidemic model using Markovian switching," Physica A: Statistical Mechanics and its Applications, vol. 462, pp. 684-704, 2016.

[15] Y. Zhao, Y. Lin, D. Jiang, X. Mao, and Y. Li, "Stationary distribution of stochastic SIRS epidemic model with standard 
incidence," Discrete and Continuous Dynamical Systems - Series B, vol. 21, no. 7, pp. 2363-2378, 2016.

[16] X. Z. Meng, S. N. Zhao, T. Feng, and T. H. Zhang, "Dynamics of a novel nonlinear stochastic SIS epidemic model with double epidemic hypothesis," Journal of Mathematical Analysis and Applications, vol. 433, no. 1, pp. 227-242, 2016.

[17] Y. Zhao, S. Yuan, and T. Zhang, "The stationary distribution and ergodicity of a stochastic phytoplankton allelopathy model under regime switching," Communications in Nonlinear Science and Numerical Simulation, vol. 37, pp. 131-142, 2016.

[18] N. Dalal, D. Greenhalgh, and X. Mao, "A stochastic model for internal HIV dynamics," Journal of Mathematical Analysis and Applications, vol. 341, no. 2, pp. 1084-1101, 2008.

[19] Z. Huang, Q. Yang, and J. Cao, "Complex dynamics in a stochastic internal HIV model," Chaos, Solitons \& Fractals, vol. 44, no. 11, pp. 954-963, 2011.

[20] C. Ji and D. Jiang, "Dynamics of an HIV-1 infection model with cell-mediated immune response and stochastic perturbation," International Journal of Biomathematics, vol. 5, no. 5, Article ID 1250039, 25 pages, 2012.

[21] D. Jiang, Q. Liu, N. Shi, T. Hayat, A. Alsaedi, and P. Xia, "Dynamics of a stochastic HIV-1 infection model with logistic growth," Physica A: Statistical Mechanics and its Applications, vol. 469, pp. 706-717, 2017.

[22] H. C. Tuckwell and E. Le Corfec, "A stochastic model for early HIV-1 population dynamics," Journal of Theoretical Biology, vol. 195, no. 4, pp. 451-463, 1998.

[23] Y. Wang, D. Jiang, T. Hayat, and B. Ahmad, "A stochastic HIV infection model with T-cell proliferation and CTL immune response," Applied Mathematics and Computation, vol. 315, pp. 477-493, 2017.

[24] R. Qesmi, J. Wu, J. Wu, and J. M. Heffernan, "Influence of backward bifurcation in a model of hepatitis B and C viruses," Mathematical Biosciences, vol. 224, no. 2, pp. 118-125, 2010.

[25] J. M. Heffernan and L. M. Wahl, "Monte Carlo estimates of natural variation in HIV infection," Journal of Theoretical Biology, vol. 236, no. 2, pp. 137-153, 2005.

[26] J. M. Heffernan and L. M. Wahl, "Natural variation in HIV infection: monte Carlo estimates that include CD8 effector cells," Journal of Theoretical Biology, vol. 243, no. 2, pp. 191-204, 2006.

[27] L. Arnold, Stochastic Differential Equations: Theory and Applications, John Wiley \& Sons, New York, NY, USA, 1974.

[28] R. Hasminskii, Stochastic Stability of Differential Equations, Sijthoff \& Noordhoff, Alphen aan den Rijn, The Netherlands, 1980.

[29] X. Mao, Stochastic Differential Equations and Applications, Horwood, Chichester, UK, 2nd edition, 1997.

[30] H. Liu, Q. Yang, and D. Jiang, "The asymptotic behavior of stochastically perturbed DI SIR epidemic models with saturated incidences," Automatica, vol. 48, no. 5, pp. 820-825, 2012.

[31] N. Ikeda and S. Watanabe, "A comparison theorem for solutions of stochastic differential equations and its applications," Osaka Journal of Mathematics, vol. 14, no. 3, pp. 619-633, 1977.

[32] Y. A. Kutoyants, Statistical Inference for Ergodic Diffusion Processes, Springer, London, UK, 2004.

[33] A. Berman and R. J. Plemmons, Nonnegative Matrices in Mathematical Sciences, Academic Press, New York, NY, USA, 1979.

[34] D. J. Higham, "An algorithmic introduction to numerical simulation of stochastic differential equations," SIAM Review, vol. 43, no. 3, pp. 525-546, 2001. 


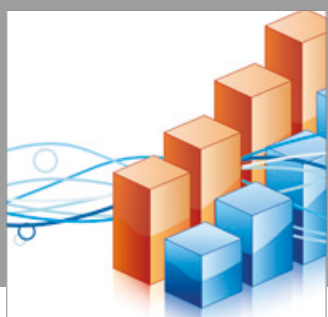

Advances in

Operations Research

vatersals

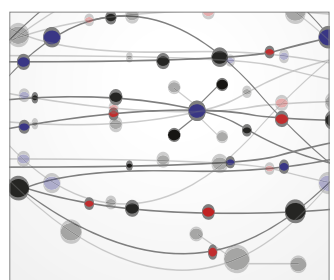

\section{The Scientific} World Journal
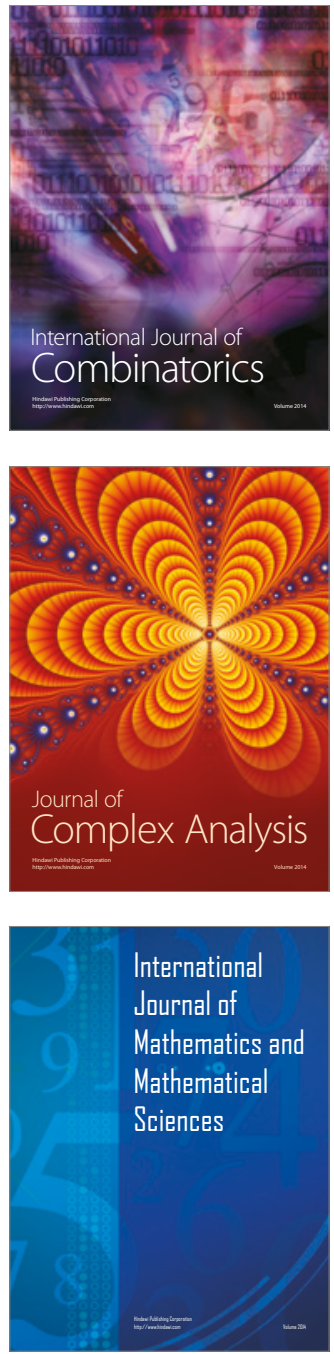
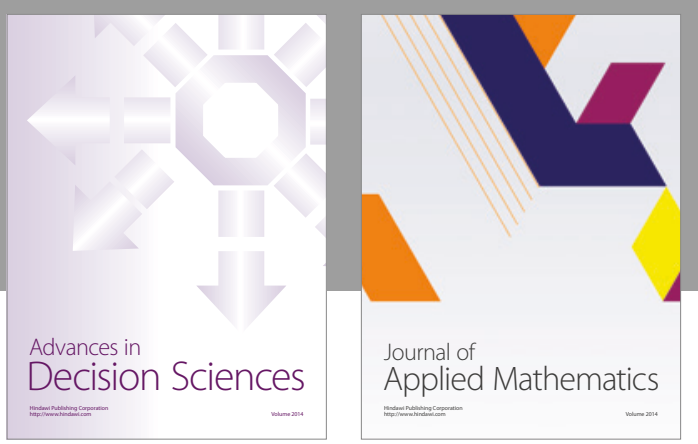

Algebra

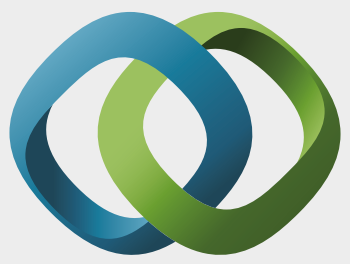

\section{Hindawi}

Submit your manuscripts at

https://www.hindawi.com
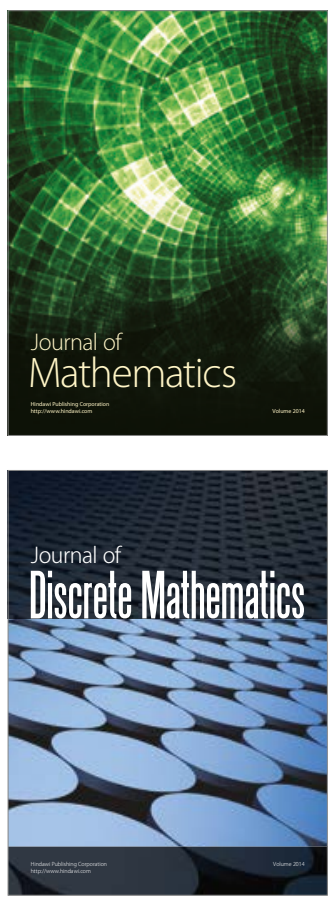

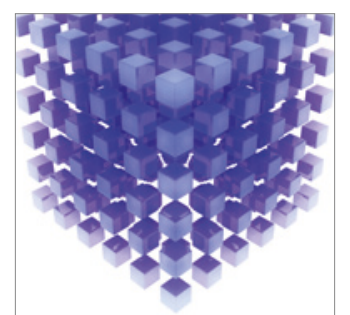

Mathematical Problems in Engineering
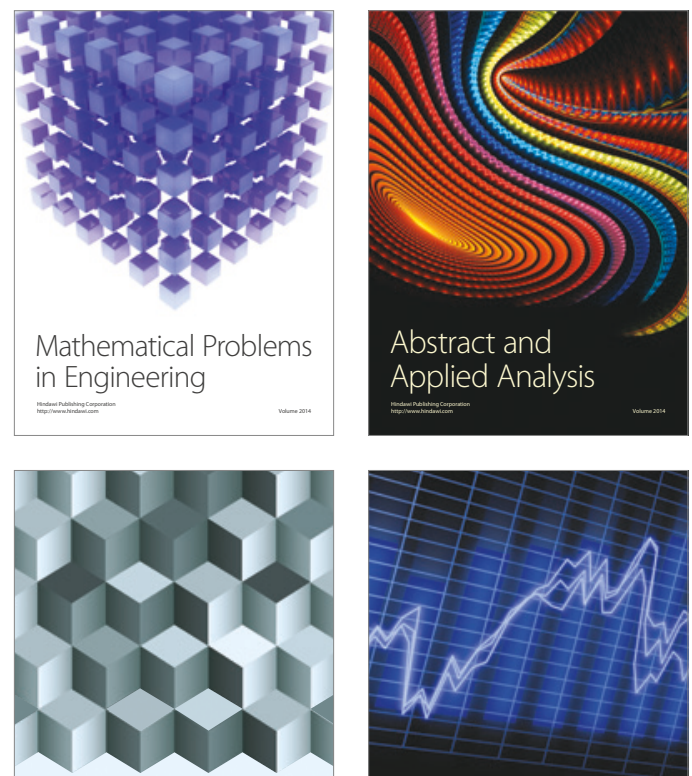

Journal of

Function Spaces

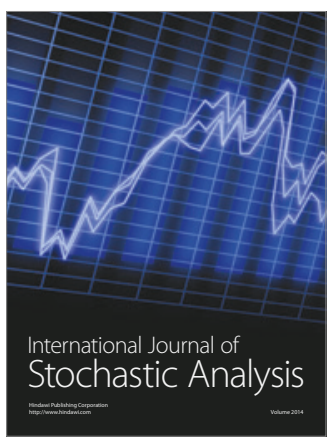

Probability and Statistics
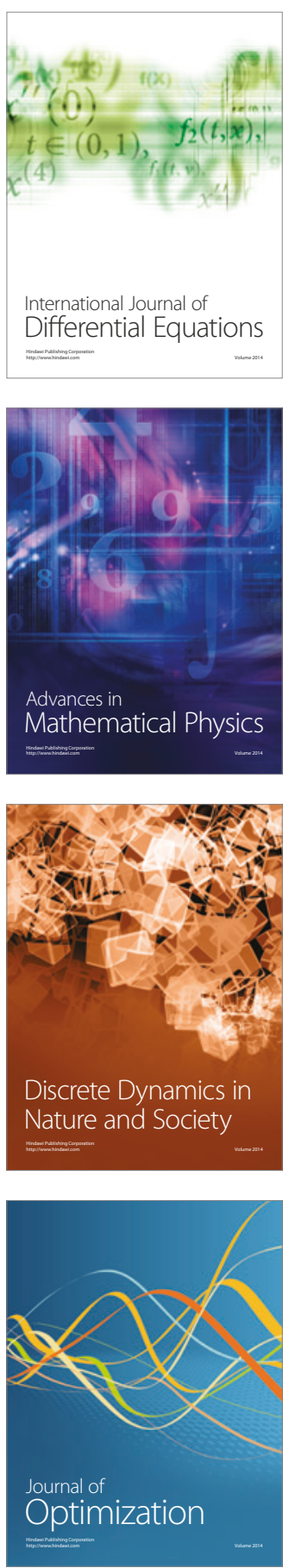